Manuscript Number: JCLEPRO-D-17-00247R1

Title: Integrating labor awareness to energy-efficient production scheduling under real-time electricity pricing: an empirical study

Article Type: Original article

Keywords: Sustainable production scheduling; Production scheduling; Optimization; Demand response; Case study; Sensitivity analysis

Corresponding Author: Mr. Xu Gong,

Corresponding Author's Institution: Ghent University/imec

First Author: Xu Gong

Order of Authors: Xu Gong; Marelies Van der Wee; Toon De Pessemier; Sofie Verbrugge; Didier Colle; Luc Martens; Wout Joseph

Abstract: With the penetration of smart grid into factories, energyefficient production scheduling has been emerged. It shifts flexible production loads to lower-priced periods to reduce energy cost for the same production task. However, the existing methods only focus on integrating energy awareness to conventional production scheduling models. They ignore the labor cost which is shift-based and follows an opposite trend of energy cost. For instance, the energy cost is lower during nights while the labor cost is higher. Therefore, this paper proposes a method for energy-efficient and labor-aware production scheduling at the unit process level. This integrated scheduling model is mathematically formulated. Besides the state-based energy model and genetic algorithm-based optimization, a continuous-time shift accumulation heuristic is proposed to synchronize power states and labor shifts. In a case study of a Belgian plastic bottle manufacturer, a set of empirical sensitivity analyses were performed to investigate the impact of energy and labor awareness, as well as the production-related factors that influence the economic performance of a schedule. Furthermore, the demonstration was performed in 9 large-scale test instances, which encompass the cases where energy cost is minor, medium, and major compared to the joint energy and labor cost. The results have proven that the ignorance of labor in existing energy-efficient production scheduling studies increases the joint energy and labor cost, although the energy cost can be minimized. To achieve effective production cost reduction, energy and labor awareness are recommended to be jointly considered in production scheduling. 


\title{
Integrating labor awareness to energy-efficient production scheduling under real-time electricity pricing: an empirical study
}

\author{
Xu Gong*, Marlies Van der Wee, Toon De Pessemier, Sofie Verbrugge, Didier Colle, Luc Martens, \\ Wout Joseph
}

Department of Information Technology, Ghent University/imec, Technologiepark 15, 9052 Ghent, Belgium

*E-mail: xu.gong@ugent.be. Tel.: +32 4886968 01. Fax: +32 93314899.

\begin{abstract}
With the penetration of smart grid into factories, energy-efficient production scheduling has been emerged. It shifts flexible production loads to lower-priced periods to reduce energy cost for the same production task. However, the existing methods only focus on integrating energy awareness to conventional production scheduling models. They ignore the labor cost which is shift-based and follows an opposite trend of energy cost. For instance, the energy cost is lower during nights while the labor cost is higher. Therefore, this paper proposes a method for energy-efficient and laboraware production scheduling at the unit process level. This integrated scheduling model is mathematically formulated. Besides the state-based energy model and genetic algorithm-based optimization, a continuous-time shift accumulation heuristic is proposed to synchronize power states and labor shifts. In a case study of a Belgian plastic bottle manufacturer, a set of empirical sensitivity analyses were performed to investigate the impact of energy and labor awareness, as well as the production-related factors that influence the economic performance of a schedule. Furthermore, the demonstration was performed in 9 large-scale test instances, which encompass the cases where energy cost is minor, medium, and major compared to the joint energy and labor cost. The results have proven that the ignorance of labor in existing energy-efficient production scheduling studies increases the joint energy and labor cost, although the energy cost can be minimized. To achieve effective production cost reduction, energy and labor awareness are recommended to be jointly considered in production scheduling.
\end{abstract}

Keywords: Sustainable production; Production scheduling; Optimization; Demand response; Case study; Sensitivity analysis

\section{Introduction}

Sustainability is a crucial factor in future production systems for manufacturing enterprises to stay competitive (May et al., 2016). It is the "development that meets the needs of the present, without compromising the ability of future generations to meet their own needs" (Brundtland Commission, 1987). When it is integrated to manufacturing enterprises, all dimensions of the triple bottom line should be followed: the economic, environmental and social dimension (Gimenez et al., 2012). Recently, production planning and scheduling have shown up as a promising industrial demand response approach for sustainable production (Giret et al., 2015).

Production scheduling is the allocation of available production resources to jobs/tasks, aiming to optimize one or more criterion, while satisfying production constraints, such as due date and operation sequence. Only recently, it has considered industrial energy consumption, which creates tangible added value.

From an economic perspective, energy-efficient production scheduling reduces the energy cost, under a volatile energy price from the deregulated electricity markets (Merkert et al., 2015). For many power-intensive industries, the electricity cost accounts for 10$50 \%$ of the final product cost (Hadera and Harjunkoski, 2013). Therefore, the potential to save energy cost remains considerable.

From an environmental perspective, energy-efficient production scheduling decreases greenhouse gas (GHG) emissions, of which manufacturing processes are known as the major source (Newman et al., 2012). Some of GHG emissions are caused by unnecessary machine idling (Liu et al., 2016) and peak power consumption in the electricity grid (Gong et al., 2016a), which are solvable by production scheduling.

From a societal perspective, energy-efficient production scheduling stabilizes the electricity grid by avoiding peak demand. This secures the power supply and delivery for local residents. Moreover, optimal energy utilization and reduced GHG emissions help enterprises meet sustainability compliance and regulations, improving an enterprise's reputation for public responsibility. 
Among these added values, the economic implication is the chief decision driver in manufacturing (Diaz-Elsayed et al., 2015). The potential of energy efficiency for cost reduction remains more than significant for manufacturing processes (Zavanella et al., 2015). Therefore, this paper focuses on the economic perspective.

Despite the ongoing automation in manufacturing and the appealing economic impact of energy-efficient production scheduling described above, labor cost is still a major part of production cost and follows the opposite trend of energy cost. For instance, labor compensation is higher at night and on weekends, while energy price is lower during these periods. Consequently, simple production load shifting to lower-priced periods in literature may increase the labor cost and rise the overall production cost, going against the expected sustainability. Therefore, it is indispensable to integrate labor awareness to energy-efficient production scheduling.

Compared to our previous work (Gong et al. 2016a), this paper has threefold contributions. (1) The energy-efficient scheduling model is enhanced by introducing labor shifts and cost, machine changeovers, as well as multiple idle modes. (2) A continuous-time shift accumulation heuristic is proposed to synchronize power states and labor shifts, as necessary part of the solution algorithm. (3) An empirical study is performed in a Belgian plastic bottle manufacturer. Extensive sensitivity analyses revealed a new understanding of energy-efficient production scheduling: energy and labor cost should be jointly considered to reduce the production cost.

The reminder of this paper is organized as follows. Sect. 2 gives the literature review and three research questions. Sect. 3 describes the energy-efficient and labor-aware production scheduling problem at the unit process level. Sect. 4 presents the solution algorithm. Sect. 5 introduces the empirical data from a Belgian plastic bottle manufacturer as case study. Sect. 6 explains the extensive sensitivity analysis results. Sect. 7 performs discussions. Sect. 8 draws conclusions.

\section{Literature review}

The shop floor configurations encompass single-machine, parallel-machine, (hybrid) flow-shop, and (flexible) job-shop (Branke et al., 2016). While each configuration has accumulated many production scheduling studies, energy efficiency has only been considered in recent years, with the emerging penetration of industrial demand response (Gahm et al., 2016).

\subsection{Energy-efficient production scheduling}

The powering-on/-off mechanism is an intuitive idea to enhance energy efficiency via production scheduling. It prevents machines from consuming energy when there are no active production jobs. This idea was first described in (Mouzon et al., 2007). Furthermore, a multi-objective genetic algorithm was utilized to minimize energy consumption and total completion time of a single machine (Yildirim and Mouzon, 2012). In addition to reducing non-cutting energy consumption, Hu et al. (2017) characterized the machining energy of machine tools. They minimized the joint non-cutting and cutting energy by sequencing the feature processing order of a part. Despite these efforts, the economic impact is vague, since energy consumption was not linked to the energy cost.

Shrouf et al. (2014) considered the volatile electricity price from the spot market in a single-machine scheduling model. Production loads were shifted to low-priced periods. However, a lack of job sequencing capability locks the energy cost saving potential of this idea. The authors further proposed to use Internet-of-Things (IoT) technologies for industrial energy management (Shrouf and Miragliotta, 2015), but gave no implication on how to link empirical energy data to the scheduling model.

Gong et al. (2016a) filled these gaps. Finite state machines (FSMs, or automata) were utilized to build an energy model whose power profiles were extracted from measurements. Job sequencing and reactive rescheduling upon disruptions during the execution of a schedule were also introduced in the scheduling model. The energy-cost-effectiveness was validated on a surface grinding process, and further demonstrated with various electricity pricing schemes (Gong et al., 2015), including time-of-use pricing (ToUP), realtime pricing (TRP), and critical peak pricing (CPP). Numerical experiments showed that a higher electricity cost saving ratio is contributed by prolongation of makespan. To specifically reduce the energy cost under ToUP, a greedy insertion heuristic was proposed in (Che et al., 2016) for a single machine scheduling model, such that it yielded high-quality solutions within 10 sec even for the instance with 5000 jobs. Fang et al. (2016) further investigated the same scheduling problem under the cases of uniform and scalable machine speeds.

Energy-efficient production scheduling can be found in the other shop floor configurations, though most of them are not explicitly linked to the energy cost. A parallel machine scheduling problem was investigated in (Li et al., 2016). Machines differ in energy consumption and discharged pollutants. The energy cost and pollutant clean-up cost were modeled as hard constraints, while the objective was to minimize the makespan. Zhang et al. (2014) studied a flow shop scheduling problem under ToUP electricity tariffs. They revealed the trade-off between reducing electricity cost and decreasing $\mathrm{CO}_{2}$ emissions. A hybrid flow shop floor configuration 
Table 1

Literature analysis of recent energy-efficient production scheduling methods

\begin{tabular}{|c|c|c|c|c|c|c|c|c|c|}
\hline \multirow{2}{*}{ Reference } & \multirow{2}{*}{ Shop floor configuration } & \multicolumn{2}{|c|}{ Energy model } & \multicolumn{2}{|c|}{ Labor model } & \multicolumn{2}{|c|}{ Optimization objective } & \multicolumn{2}{|c|}{ Problem size* } \\
\hline & & Assumed & Empirical & Shift & Personnel & Economic & Non-economic & Small & Large \\
\hline Mouzon et al. (2007) & Single-machine & $\sqrt{ }$ & & No & No & & $\sqrt{ }$ & $\sqrt{ }$ & \\
\hline Yildirim and Mouzon (2012) & Single-machine & $\sqrt{ }$ & & No & No & & $\sqrt{ }$ & & $\sqrt{ }$ \\
\hline Shrouf et al. (2014) & Single-machine & $\sqrt{ }$ & & No & No & $\sqrt{ }$ & & $\sqrt{ }$ & \\
\hline Gong et al. (2016a) & Single-machine & & $\sqrt{ }$ & No & No & $\sqrt{ }$ & & & $\sqrt{ }$ \\
\hline Che et al. (2016) & Single-machine & $\sqrt{ }$ & & No & No & $\sqrt{ }$ & & & $\sqrt{ }$ \\
\hline Li et al. (2016) & Parallel-machine & $\sqrt{ }$ & & No & No & & $\sqrt{ }$ & $\sqrt{ }$ & \\
\hline Zhang et al. (2014) & Flow-shop & $\sqrt{ }$ & & No & No & $\sqrt{ }$ & $\sqrt{ }$ & $\sqrt{ }$ & \\
\hline Luo et al. (2013) & Hybrid flow-shop & $\sqrt{ }$ & & No & No & $\sqrt{ }$ & $\sqrt{ }$ & $\sqrt{ }$ & \\
\hline Liu et al. (2016) & Job-shop & $\sqrt{ }$ & & No & No & & $\sqrt{ }$ & $\sqrt{ }$ & \\
\hline Mokhtari and Hasani (2017) & Flexible job-shop & $\sqrt{ }$ & & No & No & $\sqrt{ }$ & $\sqrt{ }$ & $\sqrt{ }$ & \\
\hline He et al. (2015) & Flexible job-shop & $\sqrt{ }$ & & No & No & & $\sqrt{ }$ & $\sqrt{ }$ & \\
\hline Zhang et al. (2017) & Flexible job-shop & $\sqrt{ }$ & & No & No & & $\sqrt{ }$ & $\sqrt{ }$ & \\
\hline
\end{tabular}

*The size of optimization problems is characterized by the number of jobs and time slots in this study. The size of problems solved by dispatching rules is excluded.

was involved in (Luo et al., 2013), where the ant colony-based scheduling method shifted loads under ToUP. The electricity cost was minimized considering the trade-off with the makespan. Liu et al. (2016) studied a job shop energy-efficient scheduling problem. Energy consumption was decreased by turning off underutilized machines, accounting for the trade-off with total weighted tardiness. A flexible job-shop scheduling problem was investigated in (Mokhtari and Hasani, 2017), where the optimization objective was to minimize the total completion time, maximize the total availability of the system, and minimize total energy cost of production and maintenance operations. He et al. (2015) proposed an energy saving method in flexible job shops. This method optimizes not only the operation sequence for reducing idle energy consumption, but also the machine tool selection for decreasing the energy consumption for machining operations. As alternative method to handle unforeseen events during the execution of a schedule (Gong et al., 2016b), a dynamic game theory based two-layer scheduling method was proposed for a flexible job shop (Zhang et al., 2017). Upon a machine's active request for processes during an idle period, the real-time scheduling task pool output a schedule, optimizing the makespan, total workload and energy consumption.

Furthermore, some recent studies are observed to perform economic benefit analysis of energy-aware production planning and scheduling. Wang and $\mathrm{Li}(2014)$ combined both electricity consumption (kWh) and peak demand (kW) to calculate the electricity cost of manufacturing systems. Although $24.8 \%$ of the per-product electricity cost was predicted, the additional consideration of the human factor was highlighted as outlook, since a time-shifted schedule with extended night hours must be paid for with a premium. The authors' preliminary case study revealed that although incorporation of labor increased the energy cost by $9 \%$, it reduced the joint energy and labor cost by $12 \%$, due to the minor proportion (3\%) of energy cost in this joint cost (Gong et al., 2017). Salahi and Jafari (2016) proposed a two-dimensional energy performance measure. A sensitivity study was performed on energy-aware single- and multi-machine production planning according to the 3-year RTP and ToUP data as well as load profile data. This study found that ToUP lead to lower total economic loss and lower risk values. The interrelationships between the production target, speed change, energy consumption, and electricity cost were investigated in (Sharma et al., 2015). Using the proposed multi-objective optimization method, a manufacturing system was demonstrated to be more eco-friendly without a substantial increase in the electricity cost.

\subsection{Gaps and research questions}

Table 1 analyzes these representative studies and unveils the following gaps. Firstly, despite these emerging investigations, energy efficiency has never been jointly considered with the labor (regarding the time associated with shifts as well as the type and quantity of personnel). For instance, an 8-h shift was involved in (Mouzon et al., 2007). But it rather defined the overall scheduling time span, instead of introducing multiple continuous shifts which are not only a practical constraint but also unlock more optimization potential for energy-cost-effective load shifting. As the makespan tends to be prolonged in these scheduling methods, the number of labor shifts and the period with higher labor wage would both increase. Therefore, the reduced energy cost has a risk of being compensated by the rising labor cost. 
Secondly, empirical power data has seldom been utilized, although IoT-enabled energy monitoring has penetrated the factories to enable empirical energy awareness and energy efficiency measures (Abele et al., 2015; Shrouf and Miragliotta, 2015). The energy consumption is only an assumed constraint in a majority of these studies, such as on/off mode and unit energy consumption or cost for production operations. Consequently, this simplifies the real problem. For example, to reduce the search space for an energyefficient scheduling solution in (Luo et al., 2013), each operation was assumed to start immediately after the previous operation. This goes against the philosophy of energy-efficient production scheduling which may insert idle or off periods between operations, thereby removing many potential solutions in the search space.

Thirdly, some studies do not link energy efficiency to economic benefits to factories, although an explicit link to the cost makes the impact of energy-efficient production scheduling more tangible. Fourthly, the problem size remains small regarding the number of jobs (order of magnitude: 1) and time slots (order of magnitude: 2). Though some investigations on the single-machine shop floor configuration tried to handle a large problem size, studies on the other shop floor configurations just ignored this scalability issue.

To fill these gaps, this paper intends to integrate labor awareness to energy-efficient production scheduling and address the following questions with both empirical and large-scale test instances: 1) Will the incorporation of energy and labor awareness in a production schedule help reduce the energy cost for production? 2) Will the incorporation of energy and labor awareness in a production schedule decrease the total energy and labor cost for production? 3) What are the potential factors that will impact the total energy and labor cost, the energy cost, as well as the labor cost of an energy-efficient and labor-aware production schedule, and to which extent?

\section{Problem description}

The problem is to assign the sequence $(\pi)$ and start time $\left(S T J_{i}\right)$ of $N_{J}$ discrete production jobs, the machine power states $(s$, including the states for an optimal idle mode between two jobs), as well as personnel type ( $p t)$ at the unit process level, under a volatile electricity price, while respecting the due date $(D T)$ and the labor rule of no production on weekends.

One job contains one product type, while different jobs contain different product types. A changeover is required between jobs. The electricity price $(E P)$ varies with time slots $(D)$, but stays constant in each $D$. Energy consumption is calculated at the power state level. One day consists of $|S H|$ shift types ( $s h \in S H$, where $S H$ is the set of all shift types) and a set of shift boundary times (SBT) at which a shift switch occurs. The wage per shift $\left(W_{s h}^{p t}\right)$ varies with $s h$ and $p t$, while $p t$ varies with $s$ which is linked to machine operations or power consumption behavior.

\subsection{Objective}

Three objective functions are formulated by Eqs. (1-3): minimization of joint energy cost $(E C)$ and labor cost $(L C)$, minimization of $E C$, and minimization of $L C$, respectively. The definition of these three objectives enables the cost performance comparison among joint energy and labor awareness, sole energy awareness, and sole labor awareness in production scheduling.

$$
\begin{aligned}
& \min _{s, \pi, S T J_{i}, p t}(E C+L C) \\
& \min _{s, \pi, S T J_{i}, p t}(E C) \\
& \min _{s, \pi, S T J_{i}, p t}(L C)
\end{aligned}
$$

\subsection{Energy cost (EC) constraints}

The calculation of $E C$ is indicated by Eq. (4). It comprises three subparts: energy cost for processing jobs $\left(C J_{i}\right)$, energy cost for performing changeovers $\left(C C_{i}\right)$, and energy cost for keeping a machine in an idle mode between two jobs $\left(C I_{i}\right)$.

$$
E C=\sum_{i=1}^{N_{J}} C J_{i}+\sum_{i=1}^{N_{J}-1}\left(C C_{i}+C I_{i}\right)
$$

Eqs. (5-7) calculate these three energy cost subparts, respectively, based on time slot ( $t s$ ), the smallest scheduling time granule. The time-related variables are defined: $S T J_{i}$ and $E T J_{i}$ are the start and end time of a job, $S T S J_{i}$ and $E T S J_{i}$ are the start time and end 
time slots of a job, $S T C_{i}$ and $E T C_{i}$ are the start and end time of a changeover, as well as $S T S C_{i}$ and $E T S C_{i}$ are the start and end time slots of a changeover.

$$
\begin{aligned}
C J_{i} & =\sum_{t s=S T S J_{i}}^{E T S J_{i}} E P_{t s}\left(\beta_{t s} \sum_{t=S T J_{i}}^{E T J_{i}} P_{p}^{t} t\right) \\
C C_{i} & =\sum_{t s=S T S C_{i}}^{E T S C_{i}} E P_{t s}\left[\beta_{t s} \sum_{t=S T C_{i}}^{E T C_{i}}\left(\sum_{s \in S_{C}} P_{s}^{t} t\right)\right] \\
C I_{i} & =\left\{\begin{array}{l}
\sum_{t s=E T S C_{i}}^{S T S J_{i+1}} E P_{t s}\left[\beta_{t s} \sum_{t=E T C_{i}}^{S T J_{i+1}}\left(\sum_{s \in S \alpha_{i}} P_{s}^{t} t\right)\right], \alpha_{i} \in\left[1,2, \ldots, N_{S}\right] \\
\sum_{t s=E T S C_{i}}^{S T S J_{i+1}} E P_{t s}\left[\beta_{t s} \sum_{t=E T C_{i}}^{S T J_{i+1}}\left(\sum_{s \in S_{o}} P_{s}^{t} t\right)\right], \alpha_{i}=N_{S}+1 \\
0, \alpha_{i}=N_{S}+2
\end{array}\right.
\end{aligned}
$$

A standby mode enables a machine to keep its power consumption at a level lower than that of production, but higher than zero (without powering off). Next to this, an idle mode is defined. It includes the possibility for a machine to stay in a standby mode and to be completely powered off.

In Eq. (6), $S_{c}$ is the set of power states $(s)$ involved in a changeover. In Eq. (7), $N_{S}$ is the total number of standby modes of a machine. $\alpha_{i}$ is the machine idle mode indicator for the $i$ th job. $S \alpha_{i}$ is the set of $s$ for switching to, staying at, and recovering from the $\alpha_{i}$-th idle mode of a machine $\left(\alpha_{i} \in\left[1,2, \ldots, N_{S}\right]\right) . S_{o}$ is the set of $s$ for switching to, staying at, and recovering from off state between jobs $\left(\alpha_{i}=N_{S}+1\right)$. The case where there is no idle period between two jobs (i.e., the next job will just follow the end of the current job) is also included $\left(N_{S}+2\right)$, which of course has zero electricity cost.

For multiple standby modes, Eq. (8) enforces there must be one and only one idle mode between two adjacent jobs.

$$
\forall \alpha_{i} \wedge\left(\exists ! \alpha \in\left[1,2, \ldots, N_{S}+1, N_{S}+2\right]\right): \alpha_{i}=\alpha
$$

Eqs. (9-10) map the current time in time slots $(t s)$ to electricity pricing time slots $(D)$ before the due date $(D T)$. This mapping is frequently used in Eqs. (5-7), since energy calculation is based on $t s$ (fine-grained), while energy cost calculation is based on $D$ (coarse-grained). In this model, fined-grained energy calculation is necessary to enable energy modeling by finite state machines or FSMs (Gong et al., 2016a).

$$
\begin{aligned}
& \beta_{t s}=\left\{\begin{array}{l}
1, \text { if } t \in[t s \cdot D,(t s+1) \cdot D) \\
0, \text { otherwise }
\end{array}\right. \\
& t s=\left\lfloor\left(t-T_{s}\right) / D\right\rfloor, t \in\left[T_{s}, T_{s}+\delta t \ldots, D T-\delta t, D T\right]
\end{aligned}
$$

3.3 Labor cost (LC) constraints

$$
\begin{gathered}
L C=\sum_{s h=S T}^{D T} \sum_{p t \in P T} W_{s h}^{p t} \cdot \theta_{s h}^{p t} \\
\delta s h=24 /|S H|
\end{gathered}
$$

$L C$ is calculated by Eq. (11), and dependent on $s h$. A shift increment in hours $(\delta s h)$ is defined in Eq. (12). Within one shift (sh), once a personnel type $(p t)$ is required by an involved power state $(s)$, this $p t$ will be included in this $s h\left(\theta_{s h}^{p t}=1\right)$. Otherwise, the binary personnel occupation indicator $\theta_{s h}^{p t}$ is zero. In other words, once a person is needed sometime in a shift, this person will work during the whole shift, regardless of the actual workload. Consequently, the number of "man-shifts" is used in this model to measure the personnel resources required for a production schedule, although the cost of a man-shift may vary a bit by $p t$. As FSMs enable $s$ to make transitions step by step, this make FSMs fit quite well to determine $\theta_{s h}^{p t}$ over time. Readers are referred to (Gong et al., 2016a) for details on machine energy modeling by FSMs. 


\subsection{Timing and sequencing constraints}

The conventional job timing constraints are defined by Eqs. (7-9) in (Gong et al., 2016a).

For a changeover, its duration $\left(D C_{i}\right)$ is inserted at the end of the $i$ th job to get prepared for the $(i+1)$-th job. Thus its start time $\left(S T C_{i}\right)$ in Eq. (13) is the end time of the $i$-th job $\left(E T J_{i}\right)$. Its end time $\left(E T C_{i}\right)$ defined in Eq. (14) is the sum of $S T C_{i}$ and $D C_{i}$ if the rest of the current week (with regard to $E T J_{i}$ ) can accommodate the entire changeover, i.e., $\omega=0$ in Eq. (15). EWD in Eq. (15) is the end time of the weekday where the $i$-th job locates. Otherwise, i.e., $\omega=1$ in Eq. (15), machine off duration, a whole weekend, and machine startup duration should be additionally considered. Both job and changeover are resumable, meaning that they can be split by a weekend and the remaining part continues by following machine startup at the start of next week.

$$
\begin{gathered}
S T C_{i}=E_{i}, i \in\left[1,2, \ldots, N_{J}-1\right] \\
E T C_{i}=S T C_{i}+D C_{i}+\omega \cdot(D S D+D W E+D S U), i \in\left[1,2 \ldots, N_{J}-1\right] \\
\omega=\left\{\begin{array}{l}
0, \text { if } S T C_{i}+D C_{i} \leq E W D_{i} \\
1, \text { otherwise }
\end{array}\right.
\end{gathered}
$$

\subsection{Production resource constraints}

Eqs. (16-17) require that a sufficient accommodation period for an idle mode must be ensured between the end time of the current changeover $\left(E T C_{i}\right)$ and the start time of next job.

$$
\begin{aligned}
& \forall \alpha_{i} \in\left[1,2, \ldots, N_{S}\right]: \sum_{s \in S \alpha_{i}} D_{s} \leq S T J_{i+1}-E T C_{i} \\
& \sum_{s \in S_{O}} D_{s} \leq S T J_{i+1}-E T C_{i}, \text { if } \alpha_{i}=N_{S}+1
\end{aligned}
$$

Eqs. (18-19) define that only one job can be produced at one time and preemption is not allowed.

$$
\begin{gathered}
\operatorname{STJ}_{i}<\operatorname{ETJ}_{i}, i \in I \\
\operatorname{ETC}_{i} \leq \operatorname{STJ}_{i+1}, i \in\left[1,2, \ldots, N_{J}-1\right]
\end{gathered}
$$

A machine only has one power state at a point of time, defined in Eq. (11) in (Gong et al., 2016a).

Eqs. (20-21) enforce the machine to be powered off before both the due date and the start of the weekend within a week.

$$
\begin{aligned}
P_{s}^{T_{s}} & =0, E T J_{N_{J}}+T S D \leq D T \\
P_{s}^{t} & =0, \forall t \in \text { weekend }
\end{aligned}
$$

\section{Solution algorithms}

\subsection{Energy modeling and genetic algorithm}

When power monitoring is performed on a production machine, a set of power states can be identified by mapping the power data to the machine functionality and operational states. Each power state has an empirical power profile. A power profile comprises an average duration and a mean power level. The machine transitions between power states over time. Once an empirical energy model is built and has the volatile electricity price as its input, it can calculate the energy consumption and cost, as well as predict the power consumption behavior over time. A GA has been widely used for solving energy-production scheduling problems. Readers are referred to (Gong et al., 2016a; Liu et al., 2015; Shrouf et al., 2014) for GA implementation.

\subsection{Power state and shift coordination}

As both machine power states and labor shifts are correlated with time, dedicated coordination is needed for correct cost calculation. Multiple cases can be identified for this coordination (Fig. 1).

Fig. 1a shows the simplest case, where the current power state $(s)$ starts and ends in the current shift. Fig. 1b presents a more complex situation, where $s$ lasts so long that new shifts are needed for full accommodation. Besides, $s$ may start exactly at the end of the current shift. If the duration of $s$ is shorter than a shift, one new shift is added (Fig. 1c). Otherwise, multiple new shifts are added 


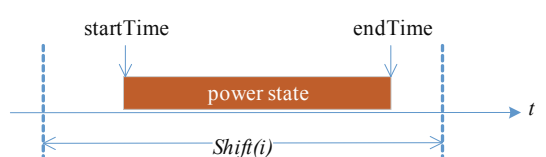

(a)

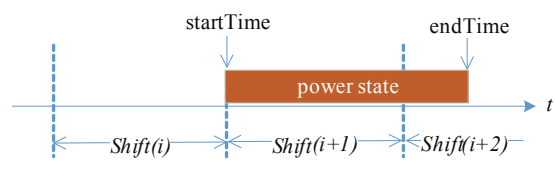

(d)

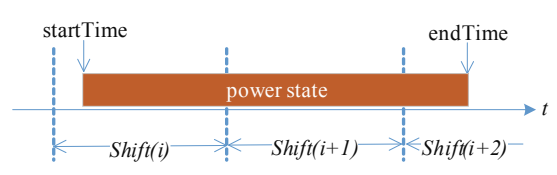

(b)

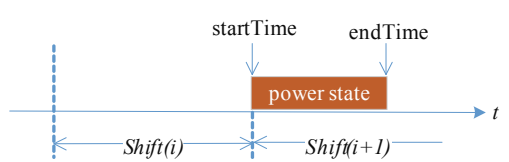

(c)

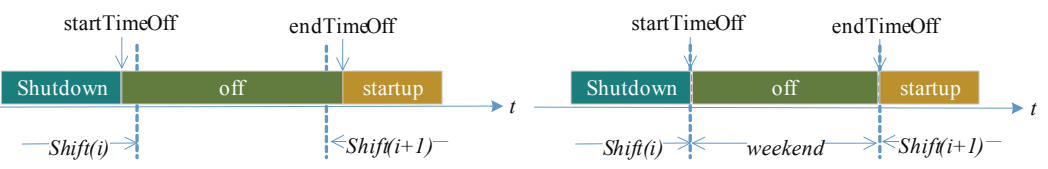

(e)

Fig. 1. Coordination of current machine power state $(s)$ and shift $($ shift $(i))$ over time.

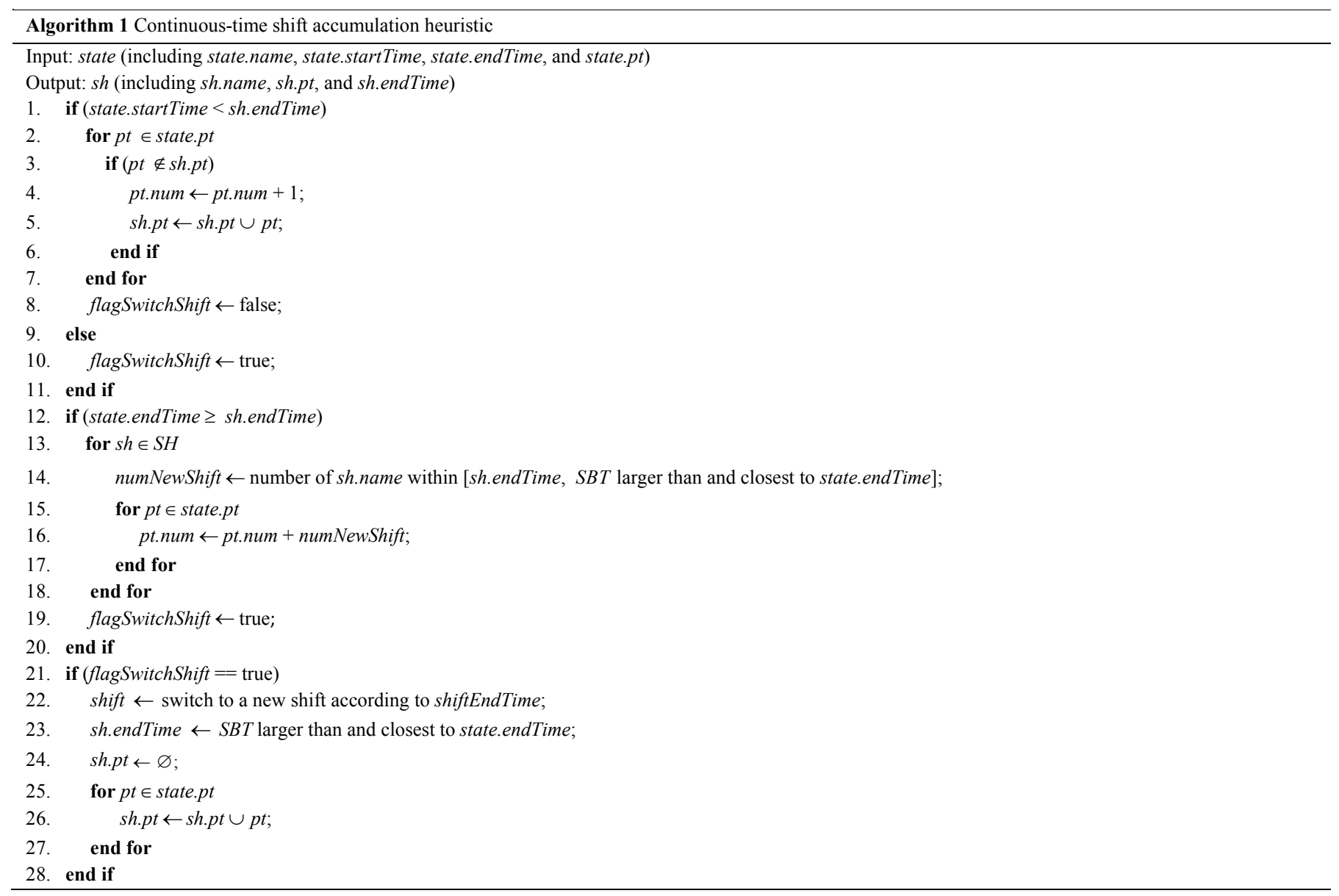

(Fig. 1d). Fig. 1e sketches a case, where a machine power-off is scheduled between two jobs. When the current job is finished, the machine may be completely shut down, stay off for an assigned period, and start up for the next job. This may need new shifts. Note that even if a shift contains one or more off periods, the entire shift duration is taken for labor cost calculation. Fig. 1f depicts a case where production is not allowed on weekends. The machine is shut down in advance, such that the end of shutdown is the start of a weekend. An ongoing job may be split by this weekend.

Based on above cases, a continuous-time shift accumulation heuristic (Algorithm 1) is proposed to accumulate the number of each personnel type with the power state transition over time. It applies to all power states except off, since no shifts are needed when a machine stays off. 
Algorithm 1 uses three global variables. The first is current shift $(s h \in S H)$, containing current time. A $s h$ includes two sub-variables: end time (sh.endTime) and personnel types already required by $s h(s h . p t)$. Sh.endTime represents a critical time point $(\in S B T)$ for a shift switch. The second global variable is current power state (state), with three sub-variable: state's start time (state.startTime), state's end time (state.endTime), and the personnel types required by state (state.pt). The third global variable is the personnel type $(p t \in P T)$, with one sub-variable: the accumulated number of $p t$ in the whole schedule (pt.num).

Algorithm 1 comprises two functional blocks. The first (lines 1-20) accumulates the number of each personnel type (pt) and determines whether to switch a shift according to state. It first judges whether state.startTime is before shift.endTime (lines 1-11). If it is not this case, the launch of state then triggers a shift switch. Otherwise, no shift switch is needed for a new shift. But it needs to check whether an additional $p t$ is introduced by state. If it is the case, this $p t$ is considered both in its accumulated number (pt.num) and in sh (sh.pt). The first functional block then decides whether state.endTime surpasses sh.endTime (lines 12-20). If it is this case (illustrated by Figs. $1 \mathrm{~b} \& 1 \mathrm{~d}$ ), it needs to account for the number of all additional shifts, which are needed for accommodating state. Besides, $s h$ has to be updated as the last new shift.

The second functional block (lines 21-29) switches the shift when necessary, and initiates a new shift. Sh.endTime is compared with $S B T$. This results a corresponding shift type, which is taken as $s h$. After initialization (lines 24-25), sh incorporates all the required personnel types of state. Note that the rest duration of sh may accommodate other states after the end of state.

\section{Empirical data and model}

A Belgian plastic bottle manufacturer was taken as empirical study. Overall, there are 17 extrusion blow molding (EBM) process lines on its shop floor, producing plastic bottles which vary from $40 \mathrm{~mL}$ to $5 \mathrm{~L}$. The entire value chain is presented in Fig. 2, where the mold changeover and blow molding process are related to production scheduling.

The overall factory data were collected through three site surveys. The power consumption of two EBM processes was monitored every $30 \mathrm{sec}$ for over one year, by installing Siemens ${ }^{\circledR}$ PAC 3200 power monitors on the three major electricity consumers: main system, hydraulic system and extruder.

\subsection{Overall factory data}

A lifecycle cost breakdown of this plant was performed to clearly link the production scheduling to all the relevant cost parts. Five lifecycle phases are presented in Fig. 3: planning, deployment, migration, operations, and teardown. The relevant cost parts are in orange.

The planning phase includes purchasing, manpower planning and long-term planning for a new EBM process. For purchasing energy, the planning phase will only account for the negotiation costs. The actual energy consumption is included in the operations phase and depends on the scheduled production. Manpower planning establishes a long-term vision for the number of employees.

The deployment phase consists of the activities needed to start the production: the necessary equipment (machines, tools and molds) should be provided and installed. The mold design and production costs (Figs. 2 \& 3) are passed on to the customer, either through a premium percentage on the yearly volume or through a dedicated payment plan. They are hence independent of production scheduling. A similar reasoning can be followed for machines and tools.

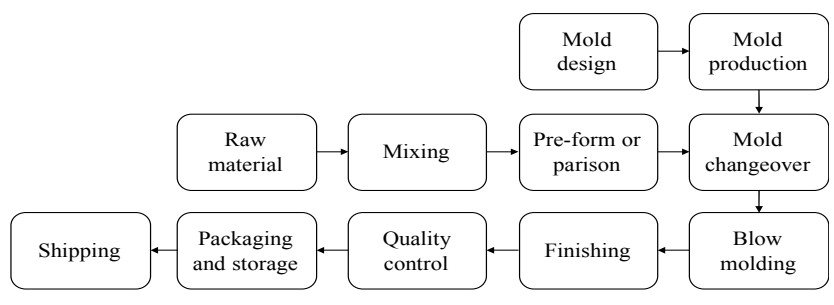

Fig. 2. Value chain of the plastic bottle manufacturer 


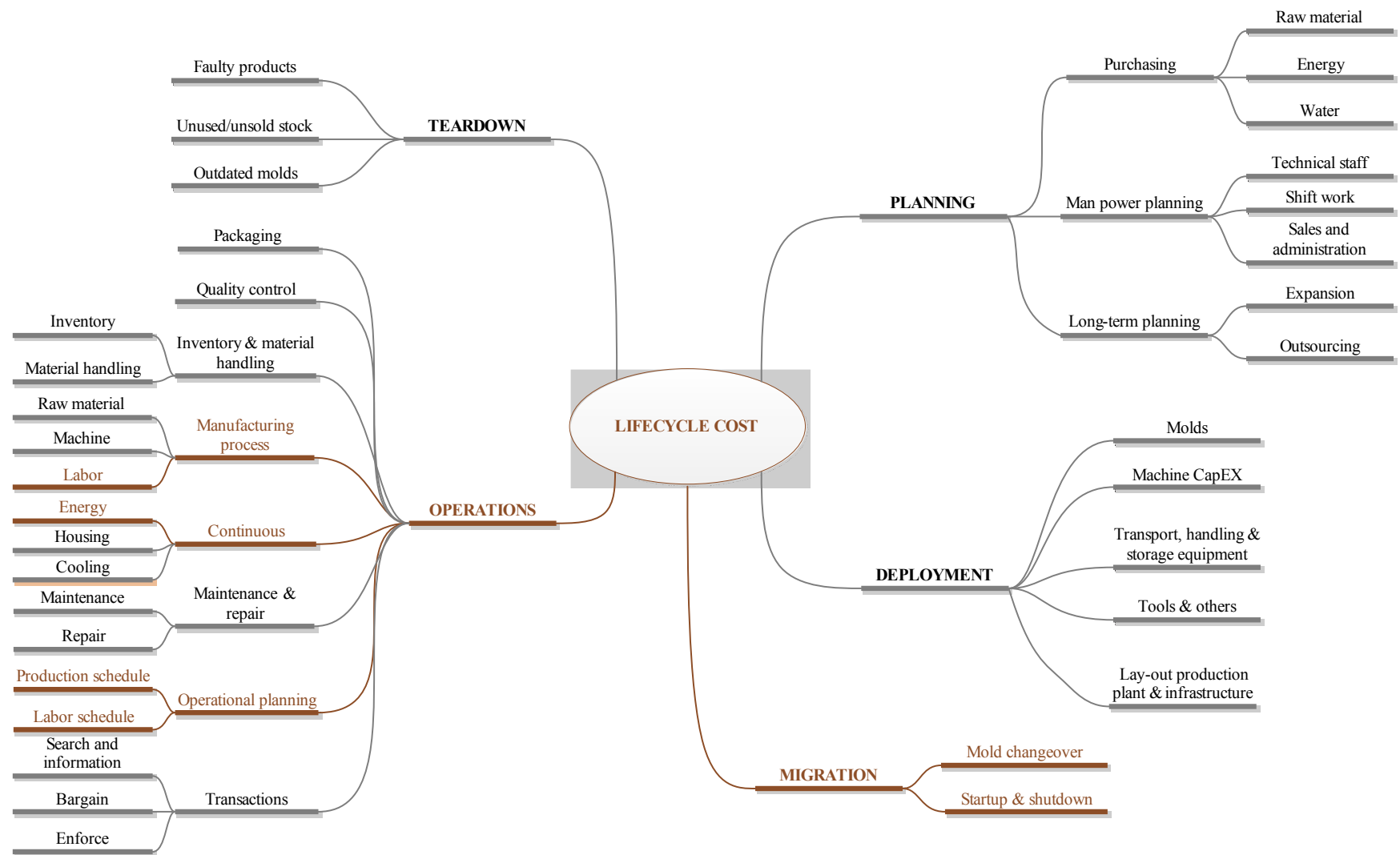

Fig. 3. Lifecycle cost breakdown of a plastic bottle manufacturer. The cost parts that are related to production scheduling are in orange. The other unrelated cost parts are in black and grey.

The migration phase denotes the costs associated with setting up a new bottle type, where the mold changeover and machine shutdown \& startup are involved. As they may influence both energy and labor costs, they are linked to production scheduling.

The operations phase includes day-to-day operational costs. The manufacturing process cost is the most important, including the raw material cost, labor cost, as well as machine CapEx and mold cost. Table 2 indicates the normalized cost types by comparing to the transparent plastic cost. Obviously, labor cost takes up an important part, demonstrating the necessity of integrating labor awareness to production scheduling for production cost minimization. Raw material cost include transparent plastic cost and color additive cost. As the production quantity (number of bottles) is prefixed, the raw material use (and hence cost) is constant, independent of production scheduling. As a result, it is logical that energy and labor cost parts are linked to production scheduling in this paper.

Table 2

Overall factory cost parts

\begin{tabular}{lc}
\hline Cost type & Normalized cost \\
\hline Transparent plastic (per kg) & 1 \\
\hline Color additives (per kg) & $3-5$ \\
\hline Machine CapEx (per hour) & 1.35 \\
\hline Packaging equipment (per hour) & 0.45 \\
\hline Packaging material (per hour) & 2.80 \\
\hline Technical staff (per hour, daytime) & 14 \\
\hline Technical staff (per hour, nighttime) & 15.5 \\
\hline Technical staff (per hour, daytime on weekends) & 19.3 \\
\hline Transport equipment (per hour) & 0.20 \\
\hline
\end{tabular}


Finally, the teardown phase represents the end-of-lifetime of EBM processes, including the processing of faulty products, unused/unsold stock and outdated molds. It is not associated with production scheduling.

\subsection{Power consumption data}

The power states identified from the measured power data include Off, Startup, Idle, Preheat, PreheatIdle, Proheat, ProheatIdle, and Production. The power profile of each state is the aggregated power profiles of these three major sub-consumers. Readers are referred to (Gong et al., 2016c) for this empirical power data and state-based energy model.

\subsection{Empirical scheduling model}

Four idle modes remain to be scheduled in-between jobs: ProheatIdle, PreheatIdle, Idle, and Off (Gong et al., 2016c). An average power profile $(2.54 \mathrm{~kW}$ during $11272 \mathrm{sec})$ was used to characterize the power consumption behavior of a bottle color changeover (Gong et al., 2016c).

As the labor aspect is considered, the empirical labor shift data were also collected. Table 3 indicates the three shifts of this plant. This factory is closed on weekends, meaning that all EBM lines have to be powered off before 6 am on Saturday and powered on again at 6 am on Monday.

Table 4 lists the personnel type required by each state of the EBM process. Specifically, ProheatIdle state has two cases. If the machine stays at ProheatIdle for powering up toward the Production state, only one operator is required. If the machine transitions from Production to ProheatIdle for a color changeover, an operator and a technician are required.

The exact labor costs cannot be disclosed due to confidentiality. But all staff is paid on an hourly basis ( $€ / \mathrm{h})$, where a bonus is paid for night shifts, with a compensation rise of $10 \%$ compared to early and late shifts. The real-time pricing (RTP) data were taken from Belpex, the Belgian electricity spot market (Belpex, 2016), where the electricity price varies every hour.

Table 3

Three shifts and working days per week of the plastic bottle manufacturer

\begin{tabular}{cllll}
\hline Early shift & Late shift & Night shift & Start of a week & End of a week \\
\hline $6 \mathrm{~h} \sim 14 \mathrm{~h}$ & $14 \mathrm{~h} \sim 22 \mathrm{~h}$ & $22 \mathrm{~h} \sim 6 \mathrm{~h}$ & $6 \mathrm{~h}$ Monday & $6 \mathrm{~h}$ Saturday \\
\hline
\end{tabular}

Table 4

Required personnel type per power state of the extrusion blow molding process

\begin{tabular}{ll}
\hline State & Required personnel (one person per type) \\
\hline Off, Startup, Idle, Preheat, PreheatIdle, Proheat & Operator \\
\hline ProheatIdle & $\begin{array}{l}\text { Operator for powering up; technician for a } \\
\text { changeover }\end{array}$ \\
\hline Production & Operator, technician, packer, quality checker \\
\hline
\end{tabular}

\section{Results}

The lifecycle cost analysis of this investigated plant (Sect. 5.1) showed that the labor cost takes up $10 \%$ of the total production cost, while the energy cost is limited to $3 \%$. The raw material cost occupies over $50 \%$, as the main cost driver.

The three optimization objectives (Eqs. 1-3) are used, respectively, with the same GA configuration on one computer (Intel i53470 CPU@3.20 GHz, 8 GB RAM). The time span is one week, corresponding to the scheduling horizon of this factory. The outcome schedule is named schedule1, schedule2 and schedule3, representing optimizing toward joint energy and labor cost (ELC), toward energy cost (EC), and toward labor cost (LC), respectively.

Random, as-early-as-possible (AEAP), and as-late-as-possible (ALAP) schedules serve as benchmarks. The random schedule is generated such that it satisfies all the formulated constraints without any optimization. The latter schedules are two rule-of-thumb scheduling strategies, which group all jobs together and starts production either as early or late as possible. These schedules have neither energy nor labor awareness. 
Table 5

Runtime and cost performance of schedules1, 2, \& 3, and a random schedule. P1: scheduling period1 (8 - 14 Aug. 2016). P2: scheduling period2 (12 - 18 Oct. 2015).

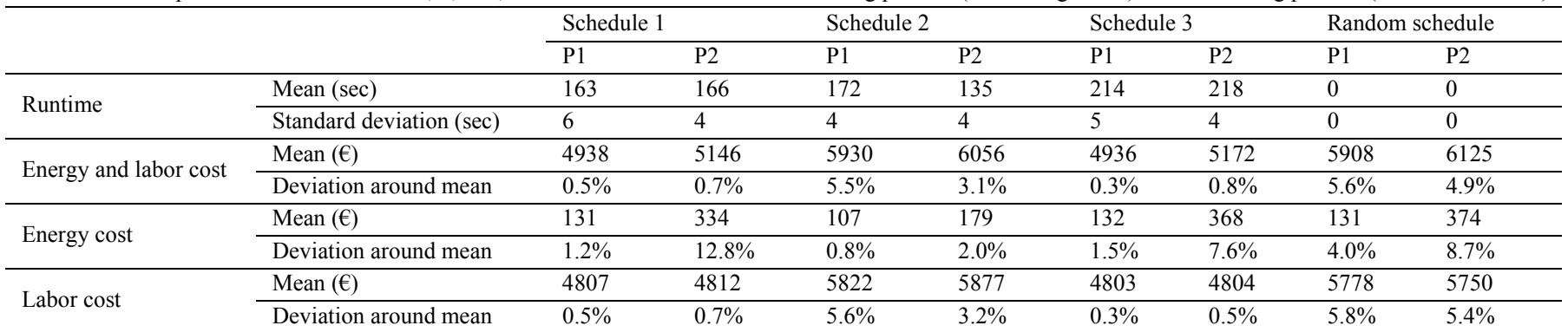

\subsection{Impact of energy and labor awareness}

The GA search for schedules1, 2, \& 3 and the random scheduling was performed 100 independent times, respectively. This experiment was repeated at two different periods: 8 - 14 Aug. 2016 (P1) when the weekly mean electricity price (MEP) is low (28 $€ / \mathrm{MWh}$ ), and 12 - 18 Oct. 2015 (P2) when the weekly MEP is high (104€/MWh). The results are indicated in Table 5.

Schedule1 and schedule3 are effective in ELC minimization. They grossly achieve the same ELC, EC and LC, in both scheduling periods. Conversely, schedule 2 and the random schedule have $17 \%$ higher ELC. The poor ELC performance of schedule 2 is explained by the minor portion of EC over the total cost (3\%).

As schedule1 optimizes LC besides EC, it avoids the situation where load shifting leads to obviously more labor shifts. For instance, shifting loads to the night may induce an additional early shift to complete the rest of production and the entire changeover. In comparison, schedule 2 incurs $21 \%$ and $22 \%$ higher LC in $\mathrm{P} 1$ and $\mathrm{P} 2$, compared schedules $1 \& 3$, respectively.

Nonetheless, schedule 2 is effective in EC minimization. It achieves the smallest variation in EC $(0.8 \%$ in $\mathrm{P} 1$ and $2.0 \%$ in $\mathrm{P} 2$, Table 5). Compared to schedules $1 \& 3$, it reduces EC by $20 \%$ and $50 \%$ in $\mathrm{P} 1$ and $\mathrm{P} 2$, respectively. Besides, its impact of EC minimization rises when the MEP increases (20\% in P1 vs. $50 \%$ in P2).

Last but not least, a schedule obtained by a single run is representative, given that the runtime and economic variation of each schedule is minor (Table 5). The following experiment results were then obtained based on one GA search.

\subsection{Impact of electricity price data}

The previous experiment was further performed at a rolling horizon of one week over 2007-2015, where full-year RTP data are available at Belpex. Consequently, $441 \times 3$ schedules were obtained. The MEP was calculated (6h Monday - 6h Saturday of the same week considering the labor shift) to indicate an averaged electricity price level per week.

\subsubsection{Economic sensitivity to electricity prices}

Fig. 4 shows the correlation between the cost parts (ELC, EC, and LC) of each schedule and weekly MEP. The Pearson correlation coefficient (CC) and p-value are indicated in each subplot. A CC value $(\epsilon[-1,1])$ close or equal to 1,0 , and -1 indicates strong positive correlation, no correlation, and strong negative correlation between two variables, respectively. A p-value should be within 5\% to guarantee the general significance of the observed statistical behavior. A column in Fig. 4 indicates different cost part of the same schedule. A row demonstrates the same cost part of different schedules.

As demonstrated in Fig. 4, both ELC and EC of three schedules follow a positive linear relation with MEP, while LC is insensitive to MEP. More specifically, schedule1's ELC has a linear relationship with MEP (CC is 0.68$)$. This is explained by the strong positive linear correlation between EC and MEP (CC is 0.97), and non-correlation between LC and MEP (CC is 0.09). A similar phenomenon is observed in schedules $2 \& 3$, except that schedule2's ELC is weakly correlated with MEP (CC is 0.14). This weaker correlation is caused by schedule2's high LC variation, which has no correlation with MEP.

Energy awareness weakens EC's sensitivity to the electricity price, although EC of the same production still rises with increasing MEP. This is indicated by the slope of the fitted linear curve on the second row of Fig. 4. A slope value equal to $k(k>0)$ infers that for every increased one euro in the electricity price, there will be additional $k$ euro added to the corresponding cost. An analogous 

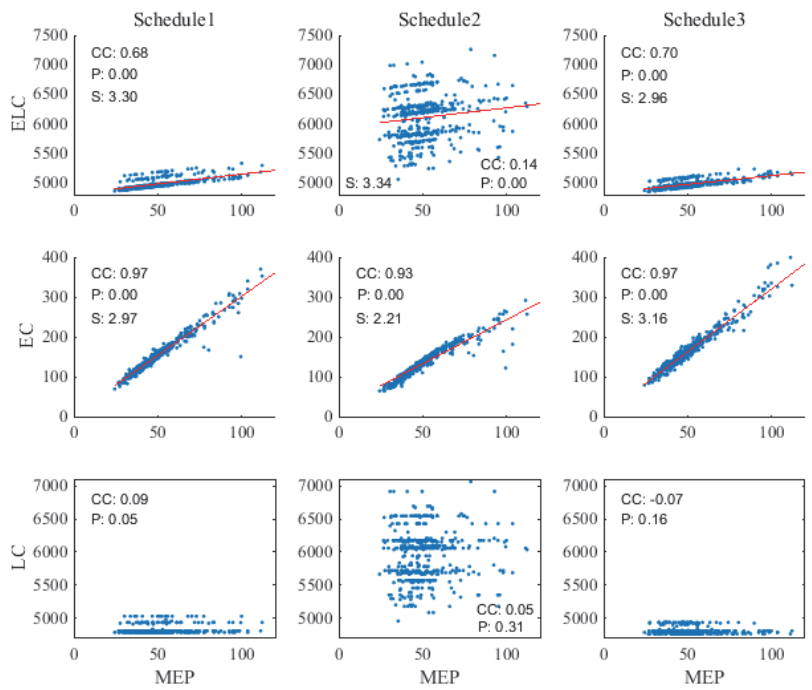

Fig. 4. Correlation between mean electricity price (MEP, $€ / M W h)$ per week and cost $(€)$ of an optimal schedule. The cost includes joint energy and labor cost (ELC), energy cost (EC), and labor cost (LC). The correlation coefficient (CC), p-value (P) and slope (S) of the fitted line are indicated in subplots.

interpretation applies to the decrease case. For EC, schedule2's slope is the smallest (2.21), while that of schedule3 is the largest (3.16). The slope of schedule1 (2.97) is closer to that of schedule3, since LC takes the major cost part such that ELC optimization is in favor of LC.

For the same reason, the slope order in the first row (Fig. 4) is inverse: schedule2 has the largest (3.34) while schedule3 has the least (2.96). Again, schedule1 stays at the intermediate level (3.30). This demonstrates that schedule1 reaches moderate sensitivity to the electricity price, in terms of both ELC and EC.

\subsubsection{Economic saving potential}

Schedule1 is taken as the target schedule, as it integrates both energy and labor awareness and exhibits no extreme sensitivity to an electricity price. Four baseline schedules are used to evaluate its economic performance (Fig. 5).

Schedule1 is significantly superior to schedule2. It is $25 \%$ superior in ELC and LC, while $12 \%$ inferior in EC (Fig. 5a). This again hints that it will impede the EC reduction performance by integrating labor and energy awareness for joint optimization. However, the loss in EC minimization is well compensated by the gain in LC minimization.

Schedule 1 and schedule 3 achieve a similar performance. This is proved by the zero average ELC saving ratio of schedule 1 compared to schedule3 (Fig. 5b). Schedule1 is 5\% superior in EC. But this gain is compensated by its $0.4 \%$ inferiority in LC. This implies that the integration of energy and labor awareness will slightly affect the LC optimization, while obtaining some gain in EC.

Schedule1 is slightly superior to the AEAP schedule. It is $2 \%$ superior in ELC (Fig. $5 \mathrm{c}$ ), which is contributed by its $3 \%$ gain in LC, despite its 5\% inferiority in EC. This demonstrates the effectiveness of schedule1 in LC minimization by shift compression. Schedulel's inferiority in EC is first of all explained by AEAP schedule's compact production without idling time between jobs. Besides, the integration of labor awareness in schedule1 impedes its EC minimization.

Schedule1 is obviously superior to the ALAP schedule. It is 3\% superior in ELC and LC, with equal performance in EC (Fig. 5d). This equal EC performance can be explained by the two aforementioned reasons.

\subsection{Impact of weekend production}

For the investigated factory, the wage of each personnel type at early and late shifts increases by $36 \%$ on weekends, while that at night shifts stays the same. All the other data and configurations remained the same, except that the weekly MEP was calculated from 6h Monday of a week to 6h Monday of the next week. The statistics are illustrated in Fig. 6.

Overall, with enabled weekend production, the cost saving potential of schedule1 evidently rises compared to the ALAP schedule, stays at the same level compared to schedule2, and slightly decreases compared to schedule3 and the AEAP schedule. 


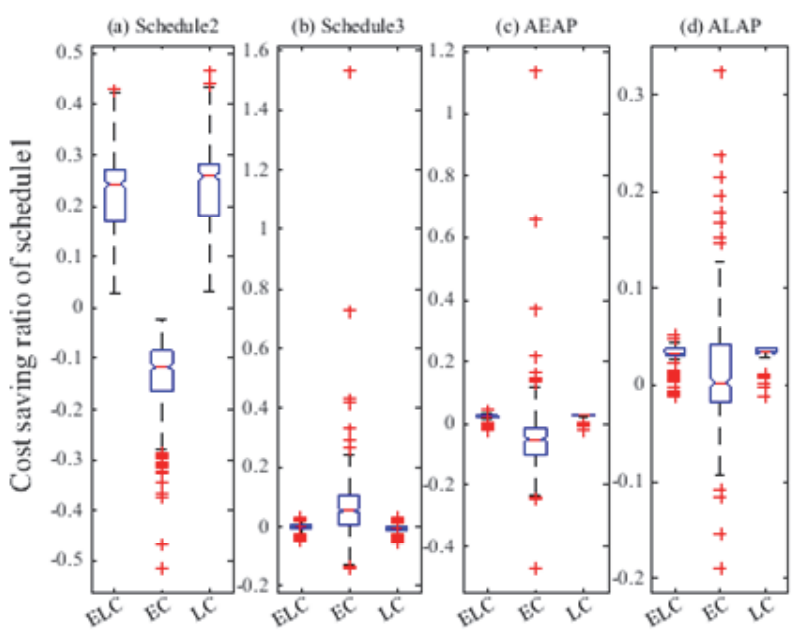

Fig. 5. Cost saving potential of schedule1 in comparison to schedule2, schedule3, as-early-as-possible (AEAP) schedule, and as-late-as-possible (ALAP) schedule. The cost parts include joint energy and labor cost (ELC), energy cost (EC), and labor cost (LC). Production is disabled on weekends.

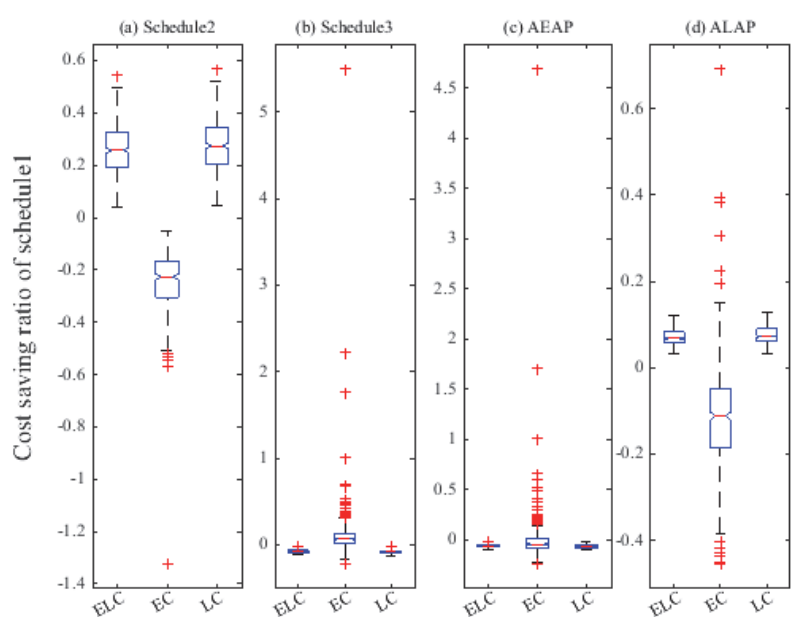

Fig. 6. Cost saving potential of schedule1 compared to schedule2, schedule3, as-early-as-possible (AEAP) schedule, and as-late-as-possible (ALAP) schedule The cost parts include joint energy and labor cost (ELC), energy cost (EC), and labor cost (LC). Production is allowed on weekends.

Compared to schedule2 (Fig. 6a), schedule1 achieves 26\% less ELC, 23\% more EC, and 27\% less LC. In comparison with the case where production is disabled on weekends (Fig. 5a), schedule1's ELC saving ratio remains the same, while the inferiority in EC and the superiority in LC are strengthened, respectively. This is explained by the missing labor awareness in schedule2. As the electricity price is lower on weekends, schedule 2 is more likely to shift production loads to weekends.

In contrast to schedule3 (Fig. 6b), schedule1 is nearly 1\% higher in ELC and LC, and 7\% lower in EC. This implies that it is more frequent for schedule1 to shift loads to the daytime (early and late shifts) on weekends. Compared to the case without weekend production (Fig. 5b), schedule1's superiority in EC and inferiority in LC are enhanced, as it assigns more weekend production to further reduce EC while slightly increasing LC.

In comparison to AEAP schedule (Fig. 6c), schedule1 is $6 \%$ higher in joint cost and LC, and 4\% higher in EC. Its inferiority in EC is explained by the two reasons elucidated in Sect. 6.2.2. This inferiority is weakened compared to the peer case (Fig. 5c), due to the lower electricity price on weekends. Additionally, as AEAP schedule has no weekend shift, schedule1 becomes also inferior in LC and joint cost, compared to this peer case. Overall, this implies that the additional EC gain on weekends is still overwhelmed by the obviously-increasing LC. 

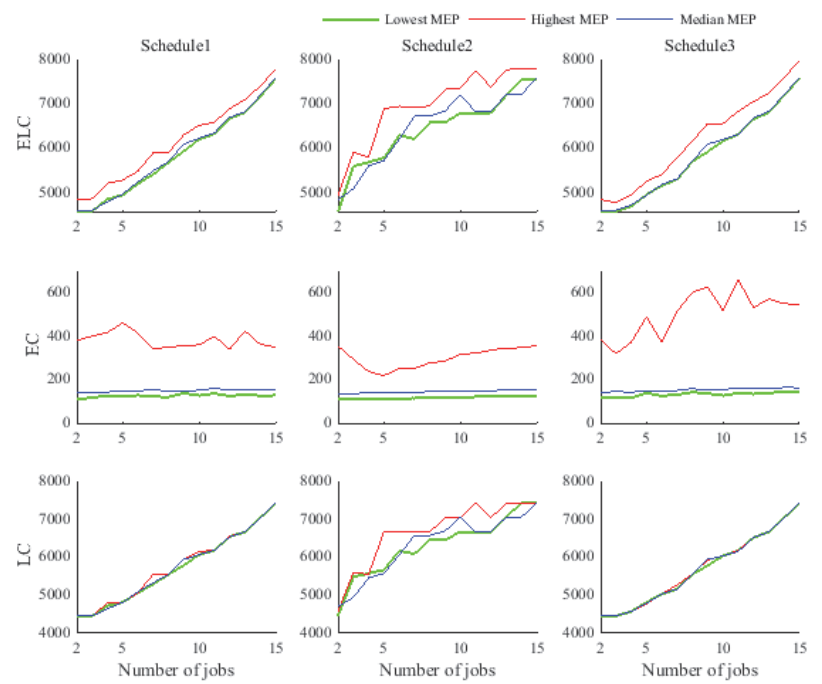

Fig. 7. Impact of the number of jobs on economic performance of schedule1, schedule2, and schedule3, respectively. The economic performance includes joint energy and labor cost (ELC), energy cost (EC), and labor cost (LC). Production is disabled on weekends.

Compared with ALAP schedule (Fig. 6d), schedule1 is 7\% lower in ELC and LC, and 11\% higher in EC. In comparison to the case without production on weekends (Fig. 5d), schedule1's superiority in ELC and LC is enhanced, since weekend shifts are always included in ALAP schedule which increases LC and subsequently ELC. However, schedule1 also becomes inferior in EC, ALAP schedule can make full use of the lower electricity price on weekends.

\subsection{Impact of production loads}

\subsubsection{Economic sensitivity to number of jobs}

The number of jobs is varied with the same load (12,500 plastic bottles) to observe the economic performance evolution of schedules1, 2 \& 3. Three one-week periods are selected from the Belpex RTP data between 2007 and 2015, such that the cases of lowest (24.32 €/MWh), highest (163.10€/MWh), and median (48.45€/MWh) weekly MEP are encompassed.

The sensitivity curves are illustrated in Fig. 7. Schedule1 exhibits the most stable economic performance.

As shown by the first column in Fig. 7, schedule1's ELC is sensitive to the number of jobs. This sensitivity is contributed by the linear relationship between LC and number of jobs, which is further explained by the increasing shift number. Comparatively, schedule1's EC is insensitive to the number of jobs. This is explained by the evidently lower power consumption during a changeover $(9.00 \mathrm{~kW})$, compared to that of bottle production $(46.35 \mathrm{~kW})$. Consequently, the increased EC for changeovers is minor compared with the EC for bottle production.

The ELC of schedule2 (second column in Fig. 7) increases with the rising number of jobs. This trend is contributed by LC, which has linear relationship with the number of jobs. The LC's sensitivity curves under three different MEP exhibit some evident difference with each other, while these almost overlap in schedules $1 \& 3$. As labor awareness is missing in schedule2, this implies that labor awareness can effectively control the LC over time, by making LC insensitive to the volatile electricity price.

Regarding schedule3 (third column in Fig. 7), the ELC and LC exhibit an analogous sensitivity as that of the other two schedules. Nevertheless, the EC under the highest MEP tends to rise with the increasing number of jobs. This is explained by the missing energy awareness in schedule3. When the electricity price is high, the EC for additional changeovers shows up, compared to the EC for fixed bottle production.

\subsubsection{Economic sensitivity to load duration}

The load duration is varied by changing the number of bottles in each job, while fixing the number of jobs at 5 . In the iterative experiment, the size of each job increases by 100 until the required accommodation reaches one week without weekend production.

The sensitivity curves are depicted in Fig. 8. Schedule1 demonstrates the most stable and predictable sensitivity to the load duration as well as the highest cost efficiency. Each schedule's ELC (first row in Fig. 8) increases with the rising load duration, fundamentally 

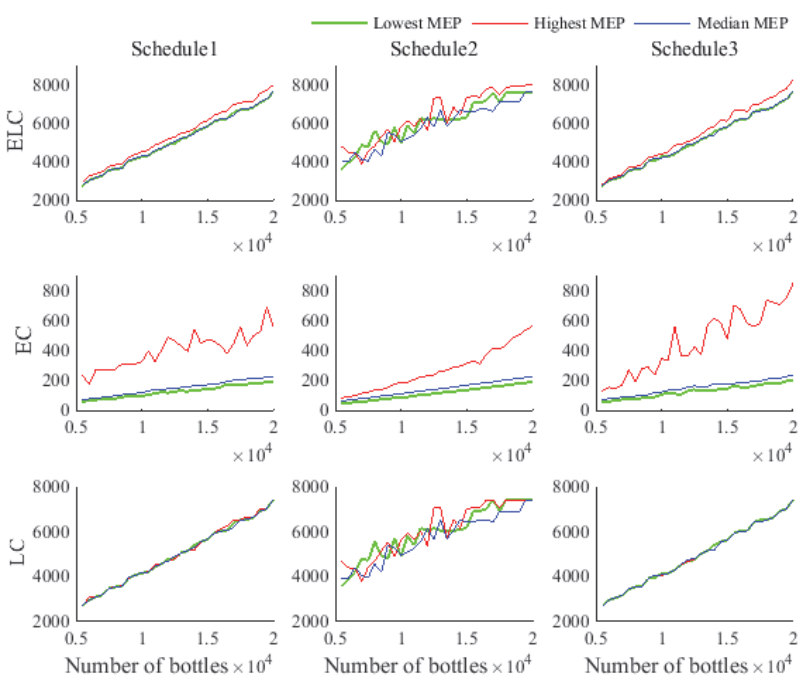

Fig. 8. Impact of load duration (i.e., number of bottles) on economic performance of schedule1, schedule2, and schedule3, respectively. The economic performance includes joint energy and labor cost (ELC), energy cost (EC), and labor cost (LC). Production is disabled on weekends.

contributed by LC.

Concerning EC (second row in Fig. 8), the sole energy awareness in schedule2 clearly contributes to the positive linear relationship between the EC and MEP. The integration of energy awareness in schedule1 is also effective, which only creates slight variation under the highest MEP. In comparison, a lack of energy awareness in schedule 3 causes significant variation under the highest MEP and an obviously higher EC under a heavy load (number of bottles is above 15,000).

Regarding LC (third row in Fig. 8), schedules1 \& 3 steadily increase with the rising number of bottles. The overlap of the three LC sensitivity curves in each of these two schedules demonstrates the effective integration of labor awareness. Comparatively in schedule2, a lack of labor awareness and the frequent load shifting enabled by sole energy awareness cause the evident variation in its LC sensitivity curves.

\subsection{Additional test instances}

Furthermore, 9 test instances were generated by scaling the power consumption (scale: 10, 100, and 1000) and the labor wage (scale: $0.8,1$, and 1.2) in the former case study, such that they encompass small, medium, and large portions of energy cost over the joint energy and labor cost. To demonstrate at a large scale, the number of time slots was 604800 (scheduling time span of one week with time granularity of $1 \mathrm{sec}$ ) and the number of jobs was 300 and 400 . For each instance, 10 runs were performed to get the average performance of schedules1, 2, \& 3, respectively. To accommodate the large number of jobs within one week, the number of plastic bottles was set as one, and the changeover time as well as the cycle time of each power state (except Production state) was set as one sec.

As indicated in Table 6, an important trend found in the former case study holds in these test instances: schedule 1 can achieve the lowest ELC. Several exceptions are observed in the instances where the power scale is 10 and labor wage scale is 1 and 1.2 (bold in Table 6). This is explained by the lower ratio of EC over ELC (EC/ELC within 9.2\%) compared to other test instances (EC/ELC above $33.1 \%$ ). However, these exceptions are minor, since schedules $1 \& 3$ have very close ELC in all these exceptions, although schedule1 has a slightly higher ELC (within 2\%).

Similar to the observation in the former case study, schedule1 never has extremely poor economic performance in contrast to the other two schedules. Schedule1 achieves an EC which is equal or close to that of schedule2 in all the test instances (Table 6), while schedule 3 has an evidently higher EC due to a lack of energy awareness. Besides, schedule1 leads to an LC close to that of schedule 3 in all the instances (Table 6), while schedule2 causes an extremely high LC (around 3 to 5 times higher compared to schedules $1 \& 3$ ) due to the missing labor awareness. Moreover, all these large-scale instances were solved within minutes (Table 6), which is a reasonable time for production scheduling. 
Table 6

Average scheduling performance in 10 runs under 9 test instances (number of time slots $=604800$, ELC: joint energy and labor cost, EC: energy cost, LC: labor cost)

\begin{tabular}{|c|c|c|c|c|c|c|c|c|c|c|c|c|}
\hline \multirow{2}{*}{$\begin{array}{l}\text { Power } \\
\text { scale }\end{array}$} & \multirow{2}{*}{$\begin{array}{c}\text { Labor } \\
\text { wage scale }\end{array}$} & \multirow{2}{*}{ Schedule } & \multicolumn{5}{|c|}{ Number of jobs $=300$} & \multicolumn{5}{|c|}{ Number of jobs $=400$} \\
\hline & & & $\operatorname{ELC}(€)$ & $\mathrm{EC}(€)$ & $\mathrm{LC}(€)$ & $\mathrm{EC} / \mathrm{ELC}$ & Runtime (sec) & $\mathrm{ELC}(€)$ & $\mathrm{EC}(€)$ & LC $(€)$ & $\mathrm{EC} / \mathrm{ELC}$ & Runtime (sec) \\
\hline \multirow{9}{*}{10} & \multirow{3}{*}{0.8} & 1 & 445 & 31 & 414 & $7.0 \%$ & 74 & 455 & 41 & 414 & $9.2 \%$ & 181 \\
\hline & & 2 & 1532 & 31 & 1501 & $2.0 \%$ & 73 & 1906 & 41 & 1865 & $2.2 \%$ & 183 \\
\hline & & 3 & 446 & 58 & 388 & $13.0 \%$ & 72 & 455 & 67 & 388 & $14.7 \%$ & 183 \\
\hline & \multirow{3}{*}{1} & 1 & 548 & 31 & 517 & $5.7 \%$ & 73 & 559 & 42 & 517 & $7.5 \%$ & 182 \\
\hline & & 2 & 2034 & 31 & 2003 & $1.5 \%$ & 75 & 2103 & 42 & 2061 & $2.0 \%$ & 182 \\
\hline & & 3 & 542 & 58 & 484 & $10.7 \%$ & 74 & 551 & 67 & 484 & $12.2 \%$ & 183 \\
\hline & \multirow{3}{*}{1.2} & 1 & 652 & 31 & 621 & $4.8 \%$ & 75 & 662 & 42 & 621 & $6.3 \%$ & 185 \\
\hline & & 2 & 2545 & 31 & 2514 & $1.2 \%$ & 75 & 2716 & 41 & 2675 & $1.5 \%$ & 184 \\
\hline & & 3 & 639 & 58 & 581 & $9.1 \%$ & 74 & 648 & 67 & 581 & $10.3 \%$ & 180 \\
\hline \multirow{8}{*}{100} & \multirow{3}{*}{0.8} & 1 & 721 & 307 & 414 & $42.6 \%$ & 75 & 830 & 416 & 414 & $50.1 \%$ & 181 \\
\hline & & 2 & 1945 & 306 & 1639 & $15.7 \%$ & 75 & 1984 & 415 & 1569 & $20.9 \%$ & 181 \\
\hline & & 3 & 967 & 579 & 388 & $59.9 \%$ & 73 & 1055 & 667 & 388 & $63.2 \%$ & 180 \\
\hline & \multirow[b]{2}{*}{1} & 1 & 824 & 307 & 517 & $37.3 \%$ & 75 & 933 & 416 & 517 & $44.6 \%$ & 181 \\
\hline & & 2 & 2275 & 306 & 1969 & $13.5 \%$ & 74 & 2597 & 415 & 2182 & $16.0 \%$ & 183 \\
\hline & \multirow{3}{*}{1.2} & 1 & 928 & 307 & 621 & $33.1 \%$ & 74 & 1037 & 416 & 621 & $40.1 \%$ & 183 \\
\hline & & 2 & 3004 & 306 & 2698 & $9.3 \%$ & 74 & 3015 & 415 & 2600 & $13.8 \%$ & 182 \\
\hline & & 3 & 1162 & 581 & 581 & $50.0 \%$ & 72 & 1247 & 666 & 581 & $53.4 \%$ & 183 \\
\hline \multirow{9}{*}{1000} & \multirow{3}{*}{0.8} & 1 & 3483 & 3069 & 414 & $88.1 \%$ & 74 & 4570 & 4156 & 414 & $90.9 \%$ & 184 \\
\hline & & 2 & 4602 & 3064 & 1538 & $66.6 \%$ & 73 & 5812 & 4148 & 1664 & $71.4 \%$ & 184 \\
\hline & & 3 & 6201 & 5813 & 388 & $93.7 \%$ & 73 & 7058 & 6670 & 388 & $94.5 \%$ & 183 \\
\hline & \multirow{3}{*}{1} & 1 & 3587 & 3069 & 517 & $85.6 \%$ & 73 & 4674 & 4157 & 517 & $88.9 \%$ & 180 \\
\hline & & 2 & 4943 & 3062 & 1881 & $61.9 \%$ & 73 & 6372 & 4147 & 2225 & $65.1 \%$ & 183 \\
\hline & & 3 & 6295 & 5811 & 484 & $92.3 \%$ & 73 & 7147 & 6663 & 484 & $93.2 \%$ & 184 \\
\hline & \multirow{3}{*}{1.2} & 1 & 3691 & 3070 & 621 & $83.2 \%$ & 74 & 4777 & 4156 & 621 & $87.0 \%$ & 183 \\
\hline & & 2 & 5470 & 3063 & 2407 & $56.0 \%$ & 74 & 6741 & 4149 & 2592 & $61.5 \%$ & 182 \\
\hline & & 3 & 6382 & 5801 & 581 & $90.9 \%$ & 72 & 7239 & 6658 & 581 & $92.0 \%$ & 183 \\
\hline
\end{tabular}

\section{Discussion}

Based on the research questions in Sect. 2 and sensitivity analyses in Sect. 6, we can advance the understanding of energy and labor awareness integration for sustainable production scheduling, and derive several managerial implications.

\subsection{Research question 1}

Integration of sole energy awareness to a production schedule can reduce the energy cost for production. If the electricity price becomes increasingly volatile, this contribution will be even more important. When the electricity price tends to increase, this integration can effectively slow down the rise of the energy cost. Besides, incorporation of both energy and labor awareness into a production schedule can also reduce the energy cost, although this reduction effect is relatively weaker. In contrast, integration of sole labor awareness in a production schedule will increase the energy cost due to the missing energy awareness.

\subsection{Research question 2}

Integration of sole energy awareness to a production schedule is not effective to decrease the total energy and labor cost. This ineffectiveness is amplified when the energy cost only occupies a minor part. Furthermore, labor cost is quite sensitive to shifting loads to periods with low electricity prices, especially when it takes up an important part in the total cost. The minor energy-saving cost can be easily compensated by the increased labor cost due to additional labor shifts.

Conversely, incorporation of both energy and labor awareness to a production schedule can effectively decrease the total cost. Analogously, this total cost efficiency is gained by sacrificing the energy cost efficiency. The actual trade-off would depend on the portion of energy cost and labor cost in every specific production case. 
Therefore, it is insufficient to only integrate energy awareness to a production schedule, which is the common practice in the existing energy-efficient production scheduling research. Energy and labor awareness are both indispensable for sustainable production. Especially in the cases where labor cost plays the major role, labor awareness is crucial for total cost reduction. In energyintensive production cases, energy awareness may be vital for total cost saving; but labor awareness is still fundamental for shop floor rostering.

\subsection{Research question 3}

For an energy-efficient and labor-aware production schedule, the sensitive factors that influence the joint energy and labor cost include the electricity price, the option for weekend production, the number of jobs, and the number of parts/products. Overall, this schedule demonstrates moderate sensitivity, without any economic performance gap. This robust economic performance facilitates production managers to guarantee stable cost reduction and to perform reliable cost prediction when facing various sensitive production parameters.

\subsection{Comparison with existing methods}

As identified in Sect. 2.2, the existing studies on energy-efficient production scheduling ignore the consideration of labor, which is directly associated with production load shifting under volatile energy prices. These existing scheduling methods are represented by schedule 2 in the former experiments (Sect. 6), i.e., solely with energy awareness. It has been demonstrated that schedule 2 evidently increases the labor cost as well as joint energy and labor cost, though the energy cost is minimized. Comparatively, the proposed scheduling method, which is represented by schedule1 (Sect. 6), can effectively reduce both energy and labor costs, thereby contributing to robust and competitive economic performance for production execution on the shop floor.

Moreover, compared to the small problem size in the majority of investigations, the proposed scheduling method has been proven to effectively work at a larger problem size, regarding the number of jobs and time slots. This competence is increasingly important with the rising needs for highly-mixed and low-volume production.

\section{Conclusion}

As a demand response approach to minimize energy cost, the existing energy-efficient production scheduling studies only focus on load shifting to lower-priced periods under real-time electricity pricing. They ignore the labor cost which has a trade-off relationship with the energy cost. A lack of labor awareness may significantly increase labor cost, which compensate the reduced energy cost.

To fill this gap, this paper proposes a method for integrated energy-efficient and labor-aware production scheduling at the unit process level, and analyzes the impact of energy and labor awareness on the economic performance of production schedules. Compared to existing literature, labor shifts and cost, machine changeovers, as well as multiple idle modes are considered in this integrated scheduling model. A continuous-time shift accumulation heuristic is proposed to coordinate the power state evolution and labor shift switch over time.

A case study was performed in a Belgian plastic bottle manufacturer. The rich factory data were utilized and analyzed to facilitate empirical scheduling, including the factory's life cycle cost breakdown, power consumption, and production scheduling. Using the 9year real-time pricing data from Belpex, the Belgian electricity spot market, extensive sensitivity analyses were carried out. Electricity price, weekend production, number of jobs, and production quantity turned out to be sensitive factors for the joint energy and labor cost. Compared to a schedule only with energy awareness or labor awareness, an energy-efficient and labor-aware production schedule demonstrated stable and superior economic performance, regarding energy cost, labor cost, and a sum of these two cost parts.

Moreover, similar results were obtained in the numerical experiments in 9 test instances, which encompass the cases where energy cost is minor, medium, and major compared to the joint energy and labor cost. As a conclusion, it is not sufficient to purely perform energy-efficient production scheduling as reported in the existing literature. To guarantee a minimized production cost (where energy and labor costs are important cost parts), it is recommended to jointly integrate energy and labor awareness in production scheduling.

Our future research will extend this scheduling method from a unit process to a larger industrial scale, such as multiple machines, multiple lines, and even multiple factories, so as to amplify its contribution to sustainable production.

\section{Acknowledgement}

This research was supported by the ICON-SENCOM (smart energy consumption in manufacturing) project with imec. 


\section{References}

Abele, E., Panten, N., Menz, B., 2015. Data collection for energy monitoring purposes and energy control of production machines. Procedia CIRP 29 , $299-304$. Belpex, 2016. Belgian electricity spot market. http://www.belpex.be/ (last access: 30.08 .2016 ).

Branke, J., Nguyen, S., Pickardt, C.W., Zhang, M., 2016. Automated design of production scheduling heuristics: A review. IEEE Trans. Evol. Comput. 20, 110-124. Brundtland Commission, 1987. Report of the world commission on environment and development: Our common future. http://www.un-documents.net/our-commonfuture.pdf (last access: 11.12.2016).

Che, A., Zeng, Y., Lyu, K., 2016. An efficient greedy insertion heuristic for energy-conscious single machine scheduling problem under time-of-use electricity tariffs. J. Clean. Prod. 129, 565-577.

Diaz-Elsayed, N., Dornfeld, D., Horvath, A., 2015. A comparative analysis of the environmental impacts of machine tool manufacturing facilities. J. Clean. Prod. 95, 223-231.

Fang, K., Uhan, N.A., Zhao, F., Sutherland, J.W., 2016. Scheduling on a single machine under time-of-use electricity tariffs. Ann. Oper. Res. $238,199-227$. Gahm, C., Denz, F., Dirr, M., Tuma, A., 2016. Energy-efficient scheduling in manufacturing companies: A review and research framework. Eur. J. Oper. Res. 248, 744-757.

Gimenez, C., Sierra, V., Rodon, J., 2012. Sustainable operations: Their impact on the triple bottom line. Int. J. Prod. Econ. 140, 149-159.

Giret, A., Trentesaux, D., Prabhu, V., 2015. Sustainability in manufacturing operations scheduling: A state of the art review. J. Manuf. Syst. 37, Part 1, 126-140.

Gong, X., De Pessemier, T., Joseph, W., Martens, L., 2015. An energy-cost-aware scheduling methodology for sustainable manufacturing. Procedia CIRP 29, 185190.

Gong, X., De Pessemier, T., Joseph, W., Martens, L., 2016a. A generic method for energy-efficient and energy-cost-effective production at the unit process level. J; Clean. Prod. 113, 508-522.

Gong, X., De Pessemier, T., Joseph, W., Martens, L., 2016b. A stochasticity handling heuristic in energy-cost-aware scheduling for sustainable production. Procedia CIRP 48, 108-113.

Gong, X., Pessemier, T.D., Joseph, W., Martens, L., 2016c. A power data driven energy-cost-aware production scheduling method for sustainable manufacturing at the unit process level, 2016 IEEE 21st Int. Conf. Emerging Technologies and Factory Automation (ETFA), 1-8.

Gong, X., Van der Wee, M., De Pessemier, T., Verbrugge, S., Colle, D., Martens, L., Joseph, W., 2017. Energy- and labor-aware production scheduling for sustainable manufacturing: A case study on plastic bottle manufacturing. Procedia CIRP 61, 387-392.

Hadera, H., Harjunkoski, I., 2013. Continuous-time batch scheduling approach for optimizing electricity consumption cost, in: Andrzej, K., Ilkka, T. (Eds.), Computer aided chemical engineering. Elsevier, pp. 403-408.

He, Y., Li, Y., Wu, T., Sutherland, J.W., 2015. An energy-responsive optimization method for machine tool selection and operation sequence in flexible machining job shops. J. Clean. Prod. 87, 245-254.

Hu, L., Peng, C., Evans, S., Peng, T., Liu, Y., Tang, R., Tiwari, A., 2017. Minimising the machining energy consumption of a machine tool by sequencing the features of a part. Energy 121, 292-305.

Li, K., Zhang, X., Leung, J.Y.T., Yang, S.-L., 2016. Parallel machine scheduling problems in green manufacturing industry. J. Manuf. Syst. 38, 98-106.

Liu, Y., Dong, H., Lohse, N., Petrovic, S., 2015. Reducing environmental impact of production during a rolling blackout policy - a multi-objective schedule optimisation approach. J. Clean. Prod. 102, 418-427.

Liu, Y., Dong, H., Lohse, N., Petrovic, S., 2016. A multi-objective genetic algorithm for optimisation of energy consumption and shop floor production performance. Int. J. Prod. Econ. 179, 259-272.

Luo, H., Du, B., Huang, G.Q., Chen, H., Li, X., 2013. Hybrid flow shop scheduling considering machine electricity consumption cost. Int. J. Prod. Econ. 146, 423439.

May, G., Stahl, B., Taisch, M., 2016. Energy management in manufacturing: Toward eco-factories of the future - a focus group study. Applied Energy 164, 628-638. Merkert, L., Harjunkoski, I., Isaksson, A., Säynevirta, S., Saarela, A., Sand, G., 2015. Scheduling and energy - industrial challenges and opportunities. Comput. \& Chem. Eng. 72, 183-198.

Mokhtari, H., Hasani, A., 2017. An energy-efficient multi-objective optimization for flexible job-shop scheduling problem. Comput. \& Chem. Eng. 104, 339-352. Mouzon, G., Yildirim, M.B., Twomey, J., 2007. Operational methods for minimization of energy consumption of manufacturing equipment. Int. J. Prod. Res. 45, $4247-4271$

Newman, S.T., Nassehi, A., Imani-Asrai, R., Dhokia, V., 2012. Energy efficient process planning for cnc machining. CIRP J. Manuf. Sci. Tech. 5, 127-136. Salahi, N., Jafari, M.A., 2016. Energy-performance as a driver for optimal production planning. Appl. Energy 174, 88-100.

Sharma, A., Zhao, F., Sutherland, J.W., 2015. Econological scheduling of a manufacturing enterprise operating under a time-of-use electricity tariff. J. Clean. Prod. $108,256-270$.

Shrouf, F., Miragliotta, G., 2015. Energy management based on internet of things: Practices and framework for adoption in production management. J. Clean. Prod. 100, 235-246.

Shrouf, F., Ordieres-Meré, J., García-Sánchez, A., Ortega-Mier, M., 2014. Optimizing the production scheduling of a single machine to minimize total energy consumption costs. J. Clean. Prod. 67, 197-207.

Wang, Y., Li, L., 2014. Time-of-use based electricity cost of manufacturing systems: Modeling and monotonicity analysis. International Journal of Production Economics 156, 246-259.

Yildirim, M.B., Mouzon, G., 2012. Single-machine sustainable production planning to minimize total energy consumption and total completion time using a multiple objective genetic algorithm. IEEE Trans. Eng. Manag. 59, 585-597.

Zavanella, L., Zanoni, S., Ferretti, I., Mazzoldi, L., 2015. Energy demand in production systems: A queuing theory perspective. Int. J. Prod. Econ. 170, Part B, 393400 .

Zhang, H., Zhao, F., Fang, K., Sutherland, J.W., 2014. Energy-conscious flow shop scheduling under time-of-use electricity tariffs. CIRP Ann. - Manuf. Tech. 63, 37-40.

Zhang, Y., Wang, J., Liu, Y., 2017. Game theory based real-time multi-objective flexible job shop scheduling considering environmental impact. J. Clean. Prod., accepted. 